\title{
ON ELASTIC GROWTH MODELLING OF STRAIGHT HAIR
}

\author{
KOSTAS P. SOLDATOS
}

\begin{abstract}
Certain macroscopic similarities of the nail and hair elongation mechanisms enable the mass-growth activity that takes place within a straight hair follicle to be modelled through a suitable extension of a relevant small-strain pseudoelasticity model of human nail growth [5]. Basic differences which are taken into consideration are the facts that straight hair (i) resembles the form of a cylindrical rod, rather than a plate, while (ii) its material constitution seems microscopically transversely isotropic, rather than isotropic. A complete analytical solution of the obtained governing differential equations is detailed for the case that incompressible mass-growth conditions prevail within the hair matrix. In addition to estimating displacement and stress distributions that develop within the growing matrix, and the resulting hair elongation, that solution enables prediction of a clinically observed zone of hair-fibre hardening that lies between the matrix soft tissue and the hard keratinous hair shaft. It also predicts that the longitudinal dimension of the hair matrix and that of the hair-fibre hardening zone depend on the material properties of the soft tissue of the follicle. Consideration of more advanced micro- or macro-scopic features of the hair follicle, such as layered structure or curved form, can be handled mathematically in a similar manner at the expense of analytical simplicity.
\end{abstract}

Keywords: Elastic mass-growth, Incompressible mass-growth, Linearly elastic growth, Mass-growth, Small strain growth, Straight hair elongation.

Kostas P. Soldatos

School of Mathematical Sciences, University of Nottingham, Nottingham NG7 2RD UK

Spencer Institute of Theoretical and Computational Mechanics, University of Nottingham,

Nottingham NG7 2RD UK

e-mail: kostas.soldatos@nottingham.ac.uk 


\section{Introduction}

The growth and elongation mechanism of straight hair (e.g., [2]) presents considerable similarities with, but also differences from its human nail counterpart (e.g., $[1,5])$. Nails and hair grow, for instance, at slow rates but, while geometrical features of a human nail resemble closely those of assembled flat plates [1], a straight hair specimen is more naturally thought of as an assembly of consecutively bonded cylindrical rods. Like the human nail specimen considered in [5], a specimen of a straight hair follicle (Figure 1) may accordingly be modelled as an assembly of structural components bonded together in series (Figure 2).

In either model, the first of the assembled structural components represents soft, proliferating, living tissue. That part of the implied structural assembly is distinguished by the customary term matrix. Cell differentiation and other germinal activity that take place in it are restricted within certain bounds. Strain levels remain thus necessarily small within the growing matrix which, by increasing its mass, forces the whole assembly to elongate by essentially pushing forward the last structural component. The latter is made of hard, keratinous, dead tissue and is unable to grow, but keeps elongating in an unbounded manner due to the aforementioned matrix activity. Those macroscopic similarities of the nail and hair elongation mechanisms suggest that the mass-growth activity that takes place within a straight hair follicle may become understood through a suitable extension of the existing elastic model of nail growth [5].

A relatively simple such extension begins here with the observation that, apart from the aforementioned macroscopic structural differences of nail and hair growth, a number of additional differences exist at some lower or microscopic scale. Some of those differences are mentioned in what follows with the help of Figure 1, though others are not considered for immediate implementation into the present, essentially pioneering attempt of hair growth modelling.

A principal such difference emerges by observing the electron micrograph of hair cross-section depicted in Figure 6 of [2]. This reveals the contents of keratinised cortical cells consisting of cylindrically-shaped closely-packed intermediate filaments embedded in protein matrix. The fact that those filaments are normal to the hair cross-section suggests that, although a single hair is essentially a single slender fibre, its material is itself fibre-reinforced. This observation is taken into consideration by the present hair growth model, which postulates that the unit normal to the hair cross-section defines a preferred material direction. Unlike its nail growth counterpart where the nail matrix is considered isotropic [5], the present model thus postulates that the hair matrix is made of transversely isotropic material.

Constitutive equations that account for small strain mass-growth of transversely isotropic elastic materials are accordingly derived in Section 2, which also summarises the set of basic governing equations involved in the present model. Section 3 introduces next the main features of the hair follicle as well as the manner in which the present mathematical model takes them into consideration. Particular attention is given to the mass-growth activity of the matrix before the critical time that triggers the mechanism of hair elongation is reached. That mechanism and the 
criterion that sets it off are then discussed in section 4. In a particular example application, Section 5 details a complete relevant analytical solution that is valid when incompressible mass-growth conditions prevails in the hair matrix. Section 6 outlines basic conclusions that stem from this study, including the observation that the longitudinal dimensions of the matrix and the hardening zone of hair-fibre depicted in Figure 1 seem to be closely dependent on the material properties of the soft tissue of the follicle.

\section{Anisotropic elastic mass-growth at small strain}

When mass-growth takes place under small strain, the relevant pseudo-elasticity type of constitutive equation [3] simplifies into the following [5]:

$$
\sigma_{i j}=\frac{\rho}{2 \rho_{0}} \frac{\partial W}{\partial e_{i j}}+\frac{r_{g}}{\rho_{0} \dot{u}_{k, k}} W \delta_{i j}
$$

where $\sigma_{i j}$ represent the components of stress in a Cartesian co-ordinate system $O x_{i}$, and $W$ is the strain energy function for mass-growth. Moreover, a dot denotes differentiation with respect to the time, $t$, the mass density, $\rho$, attains an initial value $\rho_{0}$ at $t=t_{0}$, the linear strains are defined in the usual manner,

$$
e_{i j}=\frac{1}{2}\left(u_{i, j}+u_{j, i}\right),
$$

in terms of the components of the displacement vector, $u_{i}$, while Latin suffices assume the values 1,2 and 3 , and a comma among suffices denotes partial differentiation. Finally, $r_{g}$, represents the rate of mass-growth, where the suffix " $g$ " does not assume numerical values and is, therefore, exempted from the implied indicial notation.

The continuity equation with growing mass,

$$
\frac{d \rho}{d t}+\rho \dot{u}_{i, i}=r_{g}
$$

relates the evolution of the density with the rate of mass-growth which is perceived as the principal cause of the observed mass-growth deformation. In the absence of body forces, the stress components are required to satisfy the quasi-static equations of motion, namely

$$
\sigma_{i j, j}=0 .
$$

The presence of $W$ in the second term of its-right-hand side makes it understood that, although (1) applies to small strain deformations, this constitutive equation is still non-linear in the strains. Although quadratic in the small strains, that term is necessarily retained in the (1) because is divided by the rate of volumetric strain, $\dot{u}_{i . i}$, which may be of the same order of magnitude with the linear strain components. 
In the present case of interest, where mass-growth is assumed transversely isotropic, the $x_{1}$-coordinate axis is aligned with the corresponding preferred material direction. This co-ordinate choice leads to the following quadratic form of the strain function $W$ for mass-growth [6]:

$$
W=\frac{\lambda}{2} e_{k k}^{2}+\mu_{T} e_{k m} e_{k m}+\alpha e_{k k} e_{11}+\frac{\beta}{2} e_{11}^{2}+\left(\mu_{L}-\mu_{T}\right) e_{1 k} e_{k 1},
$$

where the appearing elastic moduli relate to their standard engineering counterparts as follows (e.g., [4]:

$$
c_{11}=\lambda+2 \alpha+\beta+4 \mu_{L}-2 \mu_{T}, c_{12}=\lambda+\alpha, c_{22}=\lambda+2 \mu_{T}, c_{23}=\lambda, c_{66}=\mu_{L} .
$$

By virtue of (1), (5) yields thus the small strain constitutive equation of interest as follows:

$$
\begin{aligned}
\sigma_{i j}= & \frac{\rho}{\rho_{0}}\left\{\lambda e_{k k} \delta_{i j}+2 \mu_{T} e_{i j}+\alpha\left(e_{11} \delta_{i j}+e_{k k} \delta_{i 1} \delta_{j 1}\right)+\beta e_{11} \delta_{i 1} \delta_{j 1}\right. \\
& \left.+2\left(\mu_{L}-\mu_{T}\right)\left(e_{i 1} \delta_{j 1}+\delta_{i 1} e_{j 1}\right)\right\}+\frac{r_{g}}{2 \rho_{0} \dot{u}_{n, n}} \\
& \left\{\frac{\lambda}{2} e_{k k}^{2}+\mu_{T} e_{k m} e_{k m}+\alpha e_{k k} e_{11}+\frac{\beta}{2} e_{11}^{2}+\left(\mu_{L}-\mu_{T}\right) e_{1 k} e_{k 1}\right\} \delta_{i j} .
\end{aligned}
$$

There are particular cases of mass-growth where (7) is susceptible to further simplification. These refer to incompressible mass-growth as well as to certain types of slightly or moderately compressible mass-growth introduced and discussed in [5]. While material incompressibility is associated with kinematically constrained deformations in conventional elasticy, mass-growth incompressibility is not relevant to kinematic constrains. Instead, it only converts (3) into the following relationships:

$$
\rho=\rho_{0}, \frac{r_{g}}{\rho_{0} \dot{u}_{k, k}}=1
$$

The second of these relationships can be integrated with respect to time, and yield

$$
u_{k, k}=\frac{1}{\rho_{0}} \int_{t_{0}}^{t} r_{g} d t
$$

Moreover, it allows the quadratic strains to be considered negligible in (7), which then reduces to its conventional linear elasticity equivalent,

$$
\begin{aligned}
\sigma_{i j} & =\lambda e_{k k} \delta_{i j}+2 \mu_{T} e_{i j}+\alpha\left(e_{11} \delta_{i j}+e_{k k} \delta_{i 1} \delta_{j 1}\right)+\beta e_{11} \delta_{i 1} \delta_{j 1} \\
& +2\left(\mu_{L}-\mu_{T}\right)\left(e_{i 1} \delta_{j 1}+\delta_{i 1} e_{j 1}\right) .
\end{aligned}
$$

By inserting (10) into (4) and, where necessary, making use of (9), the following set of displacement equations of motion is then obtained in the case of incompressible mass-growth: 


$$
\begin{aligned}
& \mu_{T} u_{i, j j}+\left(\alpha+\mu_{L}-\mu_{T}\right) u_{1,1 i}+\left(\mu_{L}-\mu_{T}\right)\left(u_{1, j j} \delta_{i 1}+u_{i, 11}\right)+\beta u_{1,11} \delta_{i 1}= \\
& -\frac{\left(\lambda+\mu_{T}\right)}{\rho_{0}} \int_{t_{0}}^{t} \frac{\partial r_{g}}{\partial x_{i}} d t-\delta_{i 1} \frac{\left(\alpha+\mu_{L}-\mu_{T}\right)}{\rho_{0}} \int_{t_{0}}^{t} \frac{\partial r_{g}}{\partial x_{1}} d t .
\end{aligned}
$$

\section{Mathematical modelling features of straight hair growth}

Reference [2] bases its description of the hair follicle on a distinction between the "germinative" cells that surround the dermal papilla in the follicle bulb (Figure 1) and the growing activity that takes place above the bulb due to action of keratinassociated proteins. Thus, [2] introduces a microscopic description of the "living" part of the hair tissue that avoids deliberately the use of the relevant customary term "matrix". The latter term is, however, still employed here for purposes of macroscopic simplicity and for mathematical modelling convenience.

A plethora of additional macroscopic or microscopic composition features that may further be associated with a hair follicle [2] imply that a straight hair specimen may be approximated by an assembly of rods bonded in series. Figure 1 depicts, for instance, an intermediate zone of hardening, where disulphide bonding, resorption and dehydration make take place between the soft tissue of the matrix and the hard tissue of the mature hair shaft. However, Figure 2 considers only a pair of straight, circular cylindrical rods in series and, hence, disregards the slight follicle curvature shown in Figure 1.

Nevertheless, the structural assembly depicted in Figure 2 still allows this macroscopic mathematical model to take into immediate consideration the principal composition features of straight hair, namely $(i)$ its transversely isotropic nature, and (ii) the fact that it is approximately divided into a living part of soft matrix tissue and a dead part of keratinous hair shaft. The position of the specimen cross-section that separates germinal activity from fully keratinised material, namely

$$
x_{1}=\ell
$$

may thus be considered either measurable and, therefore, known or a location that needs to be determined.

As will be seen in Section 5 with an example application, the aforementioned intermediate zone of tissue hardening (Figure 1) may be incorporated mathematically within either the soft hair matrix $\left(0 \leq x_{1} \leq \ell\right)$ or the hard shaft tissue $\left(\ell \leq x_{1} \leq L\right)$ shown in Figure 2. Further growth and composition features may still be taken into consideration in the future and, hence, lead to refined version(s) of the present model. Wherever current lack of clinical evidence or information requires implementation of further assumptions, these are selected in a manner that serves mathematical simplicity.

The composite circular cylindrical rod depicted in Figure 2 is thus considered as idealisation of a straight hair specimen having, at $t=t_{0}$, uniform constant length $L$. The co-ordinate origin $O$ may be placed centrally either at the bottom or at the 
top end of the follicle bulb (Figure 2). The central axis of the rod coincides with the $O x_{1}$-axis and a generic hair cross-section is thus parallel to the $O x_{2} x_{3}$-plane. An arbitrary rotation of the co-ordinate plane $O x_{2} x_{3}$ about the $O x_{1}$-axis does not affect the mechanics of the model which thus perceives straight hair as an axially symmetric structure.

The radius of the hair shaft is considered constant at all times and is denoted with $h_{\ell} \equiv h_{L}$. This is equal to the measurable and, hence, known radius of the visible end of the hair, so that

$$
\left.h\right|_{\substack{t \geq t_{0} \\ x_{1} \geq \ell}}=h_{0}(\ell)=h_{\ell} \equiv h_{L},
$$

where the known smooth function $h_{0}\left(x_{1}\right)$ may be chosen to imitate the initial shape of the boundary curve of the matrix part of the specimen. That function may accordingly be defined through the initial condition

$$
\left.h\right|_{\substack{t=t_{0} \\ x_{1} \leq \ell}}=h_{0}\left(x_{1}\right),
$$

which makes clear that, due to the germinal activity of the matrix, the radius, $h$, of the shaded part of the specimen depicted in Figure 2 is considered unknown at $t>t_{0}$.

It follows that if, for simplicity, $h_{0}\left(x_{1}\right)$ is assumed constant, then (13) and (14) imply that

$$
h_{0} \equiv h_{\ell} \equiv h_{L} .
$$

The convex shape of the matrix depicted in Figure 1 suggests that, if (15) is adopted, then the shaded rod shown in Figure 2 lies within the matrix tissue and is accordingly surrounded by matrix material at all times.

The hair matrix operates within physical boundaries which do not have reasons to expand substantially. This restriction on the magnitude of the matrix deformation, also observed in nail growth, supports the postulate that the "push forward" mechanism that causes hair elongation is activated periodically, at specific critical time instances. At such a critical instant, $t=t^{c r}>t_{0}$, the strain accumulated within the grown matrix enables the relevant stress state to reach a critical intensity level that (i) negates the resultant frictions met at the lateral supports of the hair shaft, and, hence, (ii) pushes the latter forward in the $x_{1}$ direction. During that bouncing matrix action, newly grown material passes through the transitional cross-section (12) and is incorporated into the keratinised hair shaft, which is thus elongated accordingly.

When combined with the fact that hair growth is slow, these observations and postulates suggest that the small strain framework outlined in the preceding section is suitable for modelling hair matrix mass-growth, as long as the manner in which the rate of mass-growth, $r_{g}$, influences the continuity equation (3) is reasonably accounted for. In this context, reference [2] makes clear that the follicle bulb is a stronger mass producer than the upper matrix part that involves action of keratin-associated proteins. Moreover, the follicle shape (Figure 1) suggests that mass-growth decreases with distance, at least outside the bulb. New mass produc- 
tion within the matrix seems thus certainly dependent on $x_{1}$ and, possibly, on time. This may also depend on $x_{\alpha}$, where Greek indices take values 2 and 3 only. However, the small radius of the hair cross-section enables the possible dependence of $r_{g}$ on $x_{\alpha}$ to be ignored, thus suggesting that

$$
r_{g}=r_{g}\left(x_{1}, t\right)
$$

represents a set of reasonable form choices for the mass-growth rate of the hair matrix.

Because the dead part of the hair tissue is a natural continuation of its living matrix counterpart, its material may naturally be considered as approximately elastic, homogeneous and transversely isotropic. However, its considerably enhanced hardness suggests that values of Young's moduli within the shaft are much higher to those met in its neighbouring soft matrix tissue. That substantial hardness difference can then be taken to the limit, where the mature hair shaft is approximated by a rigid body which, at $t<t^{c r}$, remains completely unaffected from pressures and tractions developing within the growing matrix. Such a rigid body role of the hair shaft prevents longitudinal expansion of the matrix across the transitional plane (12) before $t=t^{c r}$, and leads to the displacement boundary condition

$$
\left.u_{1}\right|_{\substack{x_{1}=\ell \\ t<c^{c r}}}=0 .
$$

Axial symmetry allows attention to be restricted only on the upper half $\left(x_{\alpha} \geq 0\right)$ of the $O x_{1} x_{\alpha}$-plane, and its combination with the concept of continuous deformations requires that

$$
\left.u_{\alpha}\right|_{x_{\alpha}=0}=0 .
$$

at all times. Finally, validity of (13) and (14) necessitates imposition of the "corner" displacement condition

$$
\left.u_{\alpha}\right|_{\substack{x_{1}=\ell \\ x_{\alpha}=h_{\ell}}}=0
$$

The manner in which the matrix tissue operates on the transition plane (12) is practically unknown and, hence, precise specification of further boundary conditions on $x_{1}=\ell$, at $t<t^{c r}$, can only be a matter of speculation. In the same context, precise specification of further boundary conditions that may apply on the lateral $\left(x_{\alpha}=h\right)$ or the bottom $\left(x_{1}=0\right)$ boundaries of the matrix part of the specimen (Figure 2) is not possible at this stage. As is also detailed in [5], these may instead be specified in some a-posteriori manner, after a suitable potential solution of a relevant boundary value problem is sought and found. 


\section{The hair elongation criterion}

The resultant longitudinal force, $f_{1}^{c r}$, that the critical stress state is expected to negate at $t^{c r}$ is considered known or measurable. This is principally due to frictions acting on the lateral boundaries of the hair shaft and may accordingly be determined with use of mechanics rules/principles or experimental measurements which are independent of the matrix mass-growth process.

Potential availability of $f_{1}^{c r}$ implies that the following condition holds true at all times on the tissue transitional plane (12):

$$
\left.\sigma_{11}\right|_{\substack{x_{1}=\ell \\ t \leq t^{c r}}} \leq \frac{f_{1}^{c r}}{\pi h_{\ell}^{2}} .
$$

This holds in the form of an equation, namely

$$
\left.\sigma_{11}^{c r} \equiv \sigma_{11}\right|_{\substack{x_{1}=\ell \\ t=t^{c r}}}=\frac{f_{1}^{c r}}{\pi h_{\ell}^{2}},
$$

only at $t=t^{c r}$, when it dismisses and replaces the boundary condition (17).

Thus, at $t=t^{c r}$, the matrix tissue releases the elastic energy stored in it during mass-growth and bounces into a new equilibrium state. The volume of the hair matrix that crosses the transition plane (12) is thus relevant to the non-zero value that the displacement components $u_{1}$ attains at $t=t^{c r}$ on $x_{1}=\ell$. That volume of newly grown mass is then incorporated into the hard hair tissue, and enables the hair shaft to elongate, while the stress state relaxes within the matrix and returns to their initial levels measured at $t_{0}$.

Equation (21) is thus perceived as the hair elongation criterion. The resulting elongation of the hair specimen (Figure 2) is calculated by averaging over the transitional plane (12) the matrix longitudinal displacement, $u_{1}$, estimated at $t=t_{c r}$ on that plane. The total hair length is thus increased at $t^{c r}$ and becomes

$$
\hat{L}=L+\left.\frac{2}{h_{\ell}} \int_{0}^{h} u_{1}\right|_{\substack{x_{1}=\ell \\ t=t^{c r}}} d x_{2} .
$$

The outlined hair elongation process is then set to repeat itself indefinitely, after $L$ and $t^{c r}$ are replaced by $\hat{L}$ and $t_{0}$, respectively.

The outlined concept of periodically accumulated small strain enables the matrix part of the hair specimen to be considered un-deformed and unstressed in its initial configuration, leading thus naturally to the initial conditions

$$
\left.\mathbf{u}\right|_{t=t_{0}}=\mathbf{0},\left.\boldsymbol{\sigma}\right|_{t=t_{0}}=\mathbf{0} .
$$

Moreover, the notation employed in (17) and (21) implies that $u_{1}$ suffers a spatial jump discontinuity at $t^{c r}$.

However, uniqueness of linear elasticity solutions suggests that the analysis outlined in the preceding sections is unable to predict another displacement field, say 


$$
\hat{\mathbf{u}} \neq\left.\mathbf{u}\right|_{t=t^{c r}}
$$

which, for an explicitly given form of the mass-growth rate (16), (i) matches precisely the stress field implied in (20) and, at the same time, (ii) violates the alternative boundary condition (17). It follows that description of the implied matrix bouncing effect should involve additional considerations that include some concept of approximation. The example application discussed in the subsequent section clarifies further this issue. This also elucidates the manner in which the intermediate zone of hardening shown in Figure 1 is mathematically incorporated within either the soft hair matrix $\left(0 \leq x_{1} \leq \ell\right)$ or the hard hair shaft tissue $\left(\ell \leq x_{1} \leq L\right)$ shown in Figure 2.

\section{Application: Example of incompressible mass-growth of straight hair}

The simple mathematical choice

$$
r_{g}=g\left(1-x_{1} / \hat{\ell}\right)\left(t_{0} / t\right), g<<\rho_{0} / t_{0}, \ell<\hat{\ell}<L
$$

of the mass-growth rate of the hair matrix is consistent with (16) as well as with the observations that matrix growth is intense near, but declining away from the bulb of the hair follicle specimen shown in Figure 2.

While the role of the constant $g$ is evident in (25), the constant parameter $\hat{\ell}$ is perceived as a characteristic longitudinal length that marks the end of the zone of hair fibre hardening shown in Figure 1. Strict combination of (25c) with the nomenclature of Figure 2 may imply that the hardening zone is regarded as the initial part of the hard hair tissue. However, by replacing $\ell$ with $\hat{\ell}$ everywhere in Figure 2, that zone of hair hardening is seen to represent the ending part of the soft matrix tissue. Either or both of $\ell$ and $\hat{\ell}$ are accordingly considered as length parameters that need to be determined, while their precise physical significance within the follicle is currently considered as an issue of secondary mathematical importance.

By inserting (25) into (9), one thus obtains

$$
u_{k, k}=\frac{g t_{0}}{\rho_{0}}\left(1-x_{1} / \hat{\ell}\right) \ln \left(t / t_{0}\right)
$$

This shows that, while the rate of mass-growth decreases with time, the volumetric strain increases continually and, hence, enables the volume of soft tissue to increase in time. 


\subsection{The pre-critical stage of matrix mass-growth: $t<t^{c r}$}

Introduction of (25) into the Navier-type equations of motion (11) yields the partial differential equations

$$
\begin{gathered}
\hat{\mu}_{L} u_{1,11}+\mu_{L} u_{1 . \beta \beta}=\frac{\hat{\lambda} g t_{0}}{\hat{\ell} \rho_{0}} \ln \left(t / t_{0}\right), \\
\mu_{L} u_{\alpha, 11}+\mu_{T} u_{\alpha, \beta \beta}=-\left(\alpha+\mu_{L}-\mu_{T}\right) u_{1,1 \alpha},
\end{gathered}
$$

which are to be solved for the three unknown displacement components $u_{1}$ and $u_{\alpha}$ in an essentially consecutive manner. Here, use is made of the following effective elastic moduli:

$$
\begin{gathered}
\hat{\lambda}=\lambda+\alpha+\mu_{L}=c_{12}+c_{66}, \\
\hat{\mu}_{L}=\alpha+\beta+3 \mu_{L}-2 \mu_{T}=c_{11}-c_{12}-c_{66} .
\end{gathered}
$$

The simple form of the right hand side of (27) suggests that a solution of (27) that satisfies (26) and the boundary conditions (17), (18) and (19) can be sought in the form of displacement representations which are at most quadratic polynomials in the spatial co-ordinate parameters. Those representations are inserted directly into (27), (28), (17), (18), (19) and (26) and their coefficients, which are assumed functions of time only, are determined uniquely by making equal to zero the resulting coefficients of same mononyms of the spatial co-ordinate parameters. This process yields the relatively simple solution

$$
\begin{gathered}
u_{1}=-\frac{g t_{0}}{\rho_{0}}(1-\ell / \hat{\ell})\left(1-x_{1} / \ell\right) x_{1} \ln \left(t / t_{0}\right), \\
u_{\alpha}=-\frac{g t_{0}}{\rho_{0}}(1-\ell / 2 \hat{\ell})\left(1-x_{1} / \ell\right) x_{\alpha} \ln \left(t / t_{0}\right),
\end{gathered}
$$

and the additional relationship

$$
\left.\frac{\hat{\ell}}{\ell}=1+\frac{\hat{\lambda}}{2 \hat{\mu}_{L}}=1+\frac{c_{12}+c_{66}}{2\left(c_{11}-c_{12}-c_{66}\right.}\right) .
$$

It is thus seen that, apart from (17) and (18), the displacements satisfy automatically the additional boundary conditions

$$
\left.u_{1}\right|_{\substack{x_{1}=0 \\ t<t^{c r}}}=\left.u_{\alpha}\right|_{\substack{x_{1}=\ell \\ t<t^{c r}}}=0 .
$$

Moreover, (33) provides a connection between the pair of length parameters $\ell$ and $\hat{\ell}$, and the set of elastic moduli associated with the soft tissue part of the hair.

By considering for simplicity that the initial thickness of the matrix part of the hair specimen (Figure 2) is constant, (15) enters naturally into this application and leads to 


$$
h\left(x_{1}, t\right)=h_{\ell}+\left.u_{\alpha}\right|_{x_{\alpha}=h_{\ell}}=h_{\ell}\left\{1-\frac{g t_{0}}{\rho_{0}}(1-\ell / 2 \hat{\ell})\left(1-x_{1} / \ell\right) \ln \left(t / t_{0}\right)\right\} .
$$

This shows that the hair matrix exerts its active mass-growth role by pushing inwards the lateral boundary of the matrix part of the specimen. That pushing inwards trend is decreasing in the longitudinal direction, thus enabling the corner condition (19) and, hence, (13) to be satisfied at all times.

When combined with the left hand side of the inequality $(25 \mathrm{c}),(33)$ requires that

$$
\hat{\mu}_{L}>0
$$

or, equivalently, that

$$
\mu_{T}<\left(3 \mu_{L}+\alpha+\beta\right) / 2 .
$$

By virtue of (6), (37) may then take the alternative form

$$
c_{11}>c_{12}+c_{66},
$$

which is evidently expressed in terms of three rather than four elastic moduli. It is also noted with interest that, in the particular case of material isotropy, (38) reduces to the well-known Poissons ratio restriction $v<\frac{1}{2}$.

By virtue of (33), the displacement components (31) and, therefore, the corresponding strain components may be expressed in terms of the pair of effective elastic moduli defined in (29). However, it is more economical for the corresponding stress field to be expressed in terms of the engineering stiffnesses (6), namely

$$
\begin{aligned}
& \sigma_{11}=\frac{g t_{0}}{\rho_{0}}\left\{2\left[c_{11}(1-\ell / \hat{\ell})+c_{12}(1-\ell / 2 \hat{\ell})\right] \frac{x_{1}}{\ell}-c_{11}(1-\ell / \hat{\ell})-2 c_{12}(1-\ell / 2 \hat{\ell})\right\} \\
& \ln \left(t / t_{0}\right), \\
& \sigma_{22}=\sigma_{33}=\frac{g t_{0}}{\rho_{0}}\left\{\left[2 c_{12}(1-\ell / \hat{\ell})+\left(c_{22}+c_{23}\right)(1-\ell / 2 \hat{\ell})\right] \frac{x_{1}}{\ell}-c_{12}(1-\ell / \hat{\ell})\right. \\
& \left.-\left(c_{22}+c_{23}\right)(1-\ell / 2 \hat{\ell})\right\} \ln \left(t / t_{0}\right), \\
& \sigma_{1 \alpha}=-\frac{g t_{0} c_{66}}{\rho_{0} \ell}(1-\ell / 2 \hat{\ell}) x_{\alpha} \ln \left(t / t_{0}\right), \sigma_{23}=0 .
\end{aligned}
$$

Completeness of the model and uniqueness of the obtained linear elasticity solution require $(i)$ from the soft tissue that lies beyond the bottom boundary of the hair specimen $\left(x_{1} \leq 0\right)$ to support on that boundary $\left(x_{1}=0\right)$ the shear stresses $(41)$, and (ii) from the material that lies beyond the specimen lateral boundary $\left(\left|x_{\alpha}\right| \geq h_{\ell}\right)$ to support on that boundary $\left(\left|x_{\alpha}\right|=h_{\ell}\right)$ the normal stresses (40) and the shear stresses

$$
\left.\sigma_{1 \alpha}\right|_{x_{2}= \pm h_{\ell}}=\mp \frac{g t_{0} c_{66} h_{\ell}}{\rho_{0} \ell}(1-\ell / 2 \hat{\ell}) \ln \left(t / t_{0}\right),\left.\sigma_{23}\right|_{x_{2}=h_{\ell}}=0 .
$$


It is also noted that, regardless of the value of $\ell / \hat{\ell}<1, \sigma_{11}$ is negative and, therefore, compressive at the bottom end $\left(x_{1}=0\right)$ of the specimen. However, $\sigma_{11}$ becomes positive near the transitional plane $\left(x_{1}=\ell\right)$ where, with use (39), is seen to reach the maximum tensile value

$$
\left.\sigma_{11}\right|_{x_{1}=\ell}=\frac{g t_{0}}{\rho_{0}}\left\{c_{11}(1-\ell / \hat{\ell})-c_{12}(1-\ell / 2 \hat{\ell})\right\} \ln \left(t / t_{0}\right)>0 .
$$

This is, therefore, compressive within the hard tissue part of the transitional plane and, because is increasing with time, is set to reach a value which, at some $t=t^{c r}>$ $t_{0}$, fulfils (21) and triggers hair elongation.

\subsection{The critical stage: $t=t^{c r}$}

Combination of (21) and (43) yields

$$
t^{c r}=t_{0} \exp \left\{\frac{\rho_{0} \sigma_{11}^{c r}}{g t_{0}\left[c_{11}(1-\ell / \hat{\ell})-c_{12}(1-\ell / 2 \hat{\ell})\right]}\right\},
$$

which, if inserted into (31), yields the limits that the displacement components approach at $t^{c r}$ as follows:

$$
\begin{aligned}
\lim _{t \rightarrow t^{c r}} u_{1} & =-\frac{\sigma_{11}^{c r}}{c_{11}(1-\ell / \hat{\ell})-c_{12}(1-\ell / 2 \hat{\ell})}(1-\ell / \hat{\ell})\left(1-x_{1} / \ell\right) x_{1}, \\
\lim _{t \rightarrow t^{c r}} u_{\alpha} & =-\frac{\sigma_{11}^{c r}}{c_{11}(1-\ell / \hat{\ell})-c_{12}(1-\ell / 2 \hat{\ell})}(1-\ell / 2 \hat{\ell})\left(1-x_{1} / \ell\right) x_{\alpha} .
\end{aligned}
$$

Corresponding strain and stress distributions that develop within the matrix at $t^{c r}$ can then also be obtained in terms of $\sigma_{11}^{c r}$.

However, the limiting displacements (45) are unstable and cannot be maintained. The hair matrix will instead, bounce at $t^{c r}$ into the anticipated alternative equilibrium state described by some different displacement field, $\hat{\mathbf{u}}$. The elastic energy that the hair matrix releases at $t=t^{c r}$ through that bouncing effect is

$$
U^{c r}=\left.4 \int_{0}^{\ell} \int_{0}^{h\left(x_{1}\right)} \int_{0}^{h\left(x_{1}\right)} W\right|_{t=t^{c r}} d x_{3} d x_{2} d x_{1}=4\left(\sigma_{11}^{c r}\right)^{2} \bar{U}^{c r}\left(\lambda, \mu_{L}, \mu_{T}, \alpha, \beta, h_{\ell}, \ell, \hat{\ell}\right),
$$

where the value of the matrix part of the specimen thickness, $h\left(x_{1}\right)$, is evaluated at $t=t^{c r}$, while (5) is used for evaluation of $W$. It is noted that, because the critical stresses are proportional to $\sigma_{11}^{c r}, U^{c r}$ is necessarily proportional to $\left(\sigma_{11}^{c r}\right)^{2}$. Hence, $\bar{U}^{c r}$ may be determined analytically or numerically, as soon as the values of its arguments become available. 


\subsection{Spring-back type elongation of the hair matrix}

By receiving no resistance from the hard part of the hair tissue at $t=t^{c r}$, the strained and stressed grown matrix specimen acts as an independent elastic rod of length $\ell$ that bounces instantly into an alternative, unstrained and unstressed equilibrium state, where its radius returns back to its initial value, $h_{\ell}$, and its length increases due to the resulting additional displacement field, $\hat{\mathbf{u}}$.

In a corresponding static equilibrium situation, an initially unstressed and unstrained elastic, circular cylindrical rod having the same geometrical and material properties with those of the unstrained matrix part of the specimen may undergo an analogous displacement "jump" if $(i)$ its $x_{1}=0$ end is restrained against translation; (ii) its $x_{1}=\ell$ end is subjected to a uniform compressive stress, $-\sigma_{11}^{c r}$, that enables its stored energy to match the $U^{c r}$-level implied in (46); and (iii) is allowed to bounce back to its initial unstressed and unstrained state through instantaneous removal of the externally applied compression.

The inverse, linear elasticity problem that needs then to be solved is that of uniaxial compression of a transversely isotropic circular cylindrical rod having $(i)$ axial length $2 \ell,(i i)$ radius and material properties identical to those of the matrix part of the specimen, and (iii) both ends, $x_{1}= \pm \ell$, subjected to a uniform compression, $-\sigma_{11}^{c r}$. Due to evident symmetries, the middle cross-section of that rod, placed at $x_{1}=0$, will undergo no axial displacement. The loading conditions applied on a half of that rod, $0 \leq x_{1} \leq \ell$ say, are perceived as mechanically equivalent to those applied at $t=t^{c r}$ on the grown part of the matrix specimen. The attained displacement distribution is accordingly considered as approximating - $\mathbf{u}$ very closely.

It may readily be verified that the alternative displacement field sought is

$$
-\hat{u}_{i} \cong-\left.u_{i}\right|_{t=t^{c r}}=\left(-\sigma_{11}^{c r}\right) \alpha_{i} x_{i}
$$

where the appearing constant coefficients are as follows:

$$
\begin{gathered}
\alpha_{1}=\frac{c_{22}+c_{23}}{c_{11}\left(c_{22}+c_{23}\right)-2 c_{12}^{2}}=\frac{\left(\lambda+\mu_{T}\right)}{\left(\lambda+2 \alpha+\beta+4 \mu_{L}-2 \mu_{T}\right)\left(\lambda+\mu_{T}\right)-(\lambda+\alpha)^{2}}, \\
\alpha_{2}=\alpha_{3}=-\frac{\lambda+\alpha}{\left(\lambda+2 \alpha+\beta+4 \mu_{L}-2 \mu_{T}\right)\left(\lambda+\mu_{T}\right)-(\lambda+\alpha)^{2}} .
\end{gathered}
$$

By producing a constant strain field, (47) returns

$$
\sigma_{11}=-\sigma_{11}^{c r}
$$

as the only non-zero stress component acting throughout the compressed transversely anisotropic circular cylindrical rod. Hence, it satisfies exactly all associated boundary conditions and the equilibrium equations (4).

The strain energy associated with (47), namely 
$U^{c r}=\frac{\pi \ell h_{\ell}^{2}\left(c_{22}+c_{23}\right)}{c_{11}\left(c_{22}+c_{23}\right)-2 c_{12}^{2}}\left(\sigma_{11}^{c r}\right)^{2}=\frac{\pi \ell h_{\ell}^{2}\left(\lambda+\mu_{T}\right)\left(\sigma_{11}^{c r}\right)^{2}}{\left(\lambda+2 \alpha+\beta+4 \mu_{L}-2 \mu_{T}\right)\left(\lambda+\mu_{T}\right)-(\lambda+\alpha)^{2}}$,

is required to balance its counterpart implied in (46), thus leading to

$$
\bar{U}^{c r}\left(\lambda, \mu_{L}, \mu_{T}, \alpha, \beta, h_{\ell}, \ell, \hat{\ell}\right)=\frac{\pi \ell h_{\ell}^{2}\left(\lambda+\mu_{T}\right)}{4\left(\lambda+2 \alpha+\beta+4 \mu_{L}-2 \mu_{T}\right)\left(\lambda+\mu_{T}\right)-(\lambda+\alpha)^{2}} .
$$

This equation and (33) form then a set of two simultaneous algebraic equations for the two geometric parameters $\ell$ and $\hat{\ell}$, which can thus be determined in terms of the elastic moduli of the transversely isotopic hair matrix and the thickness, $h_{\ell}$, of the hair shaft.

Alternatively, if the geometric parameters $\ell, \hat{\ell}$ and $h_{\ell}$ are practically measurable, then only three of the five independent elastic moduli of the transversely isotropic matrix material need to be determined experimentally. The remaining two unknown elastic moduli are determined by solving simultaneously the algebraic equations (33) and (52). The spring-back displacement field, $\hat{\mathbf{u}}$, is then estimated by inserting into (48) the obtained values of the elastic moduli and is, thus, seen indirectly dependent on $\ell, \hat{\ell}$ and $h_{\ell}$.

When the internal energy (46) is released at $t=t_{c r}$, the opposite of the displacement field (47) brings the hair matrix into the aforementioned unstressed and unstrained state. Combination of (47) and (22) yields thus the hair elongation formula sought as follows:

$$
\hat{L}=L+2 \alpha_{1} \sigma_{11}^{c r} \ell
$$

where $\alpha_{1}$ is given according to (48). The outlined hair elongation process is then set to be repeated, after $t_{0}$ and $L$ are replaced by $t^{c r}$ and $\hat{L}$, respectively.

\section{Conclusions}

A most interesting result stemming from the analytical solution detailed in the preceding section is that the longitudinal dimension of both the matrix part of the hair follicle and the hardening zone of hair-fibre depicted in Figure 1 depend on the material properties of the soft tissue of the hair follicle. What is, of course, still unclear is whether that hardening zone of hair-fibre should be considered as the ending part of the hair matrix or the initial part of the mature hair shaft. This, however, is an issue that has to be resolved experimentally rather than mathematically. The outlined mathematical solution predicts that indeed such a hardening zone of hair-fibre does exist between the matrix proliferating part and the mature shaft part of a hair.

This conclusion is perceived as clear evidence of the fact that the present model enables interpretation of the hair elongation mechanism in a mathematically reliable and physically sound manner. Still though, more advanced features of the hair follicle can be considered and implemented into the model. These include, for instance, the layered structure of the hair follicle and/or its bent form shown in Figure 1, a 
more precise consideration of mass-growth rates, and the variable material properties that may prevail within the hardening hair-fibre zone, particularly in view of the clear distinction made in [2] between the activity of the germinative epithelial cells of the follicle bulb and the complex action of proteins that becomes associated with keratin intermediate filaments during keratinisation.

Several of these advanced modelling features may be considered by increasing the number of rods that form the structural assembly depicted in Figure 2, while others may require from the model to consider conditions of slight or moderate compressibility of matrix mass-growth (see also [5]). These, as well as additional potential refinements of the present model are possible, and may be handled mathematically in a similar manner at the expense of analytical convenience.

Acknowledgements Figure 1 is included in this paper with permission of the author of Reference [2], Emeritus Professor George E. Rogers, AO DSc FAA, Honorary Research Fellow, Cellular and Molecular Biology, School of Biological Sciences, University of Adelaide, Australia. The author is grateful to Professor G.E. Rogers for offering him overwhelmingly this permission. He is also grateful to Mrs Barbara Homer and Ms Krystyna Glowczewska for valuable technical assistance.

\section{References}

1. Baran, R., Dawber, R.P.R., Haneke, E., Tosti, A., Bristow, I.: A Text Atlas of Nail Disorders. 3rd Edn., Taylor \& Francis, London (2002)

2. Powell, B.C. and Rogers, G.E.: The role of keratin proteins and their genes in the growth, structure and properties of hair. In: Formation and Structure of Human Hair, Jollès, P., Zahn, H. and Höcker, H., Eds., Basel, Birkhäuser, pp. 59-148 (1997)

3. Soldatos, K.P.: Modelling framework for mass-growth. Mech. Res. Comm. 50, $50-57$ (2013)

4. Soldatos, K.P.: Foundation of polar linear elasticity. J. Elast. 114, 155-178 (2014)

5. Soldatos, K.P.: Small strain growth and the human nail. J. Elast. 124, 57 - 80 (2016)

6. Spencer, A.J.M.: Constitutive theory for strongly anisotropic solids. In: Continuum Theory of Fibre-Reinforced Materials, Spencer, A.J.M., Ed., CISM Courses and Lectures 282, pp. 1-32, Springer (1984) 


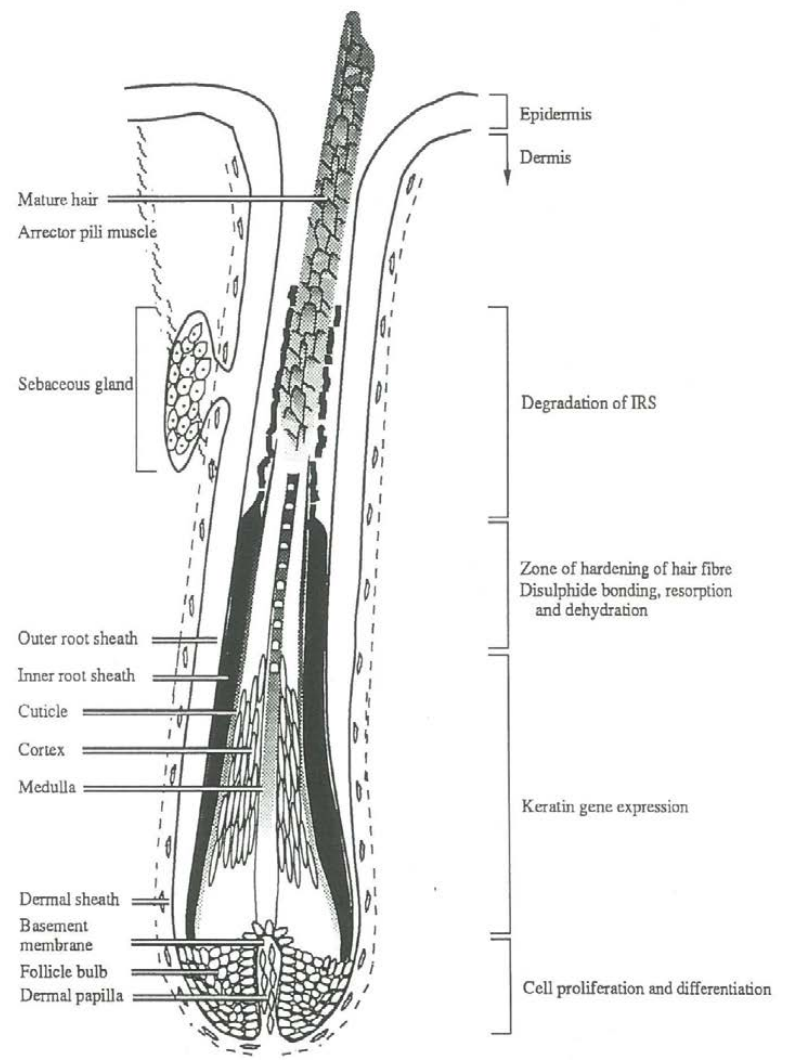

Fig. 1 Detailed schematic representation of the hair follicle outlining various features and the regions where main events of cell proliferation and keratinisation take place (reproduced from page 81 of [2] by the author's, Professor George Ernest Rogers, permission). 


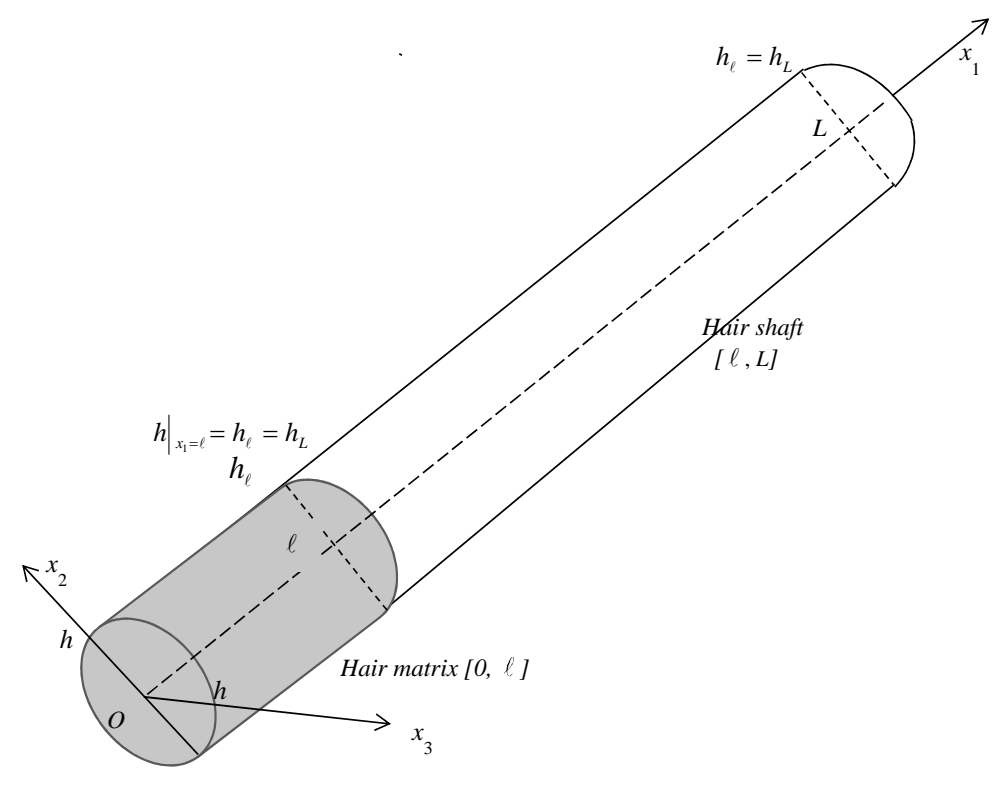

Fig. 2 Geometrical representation of a straight hair specimen in the form of two consecutive circular cylinders. The shaded (bottom) and unshaded (top) parts represent soft (germinal) and hard (keratinous) tissue material respectively. 